\title{
Expression profiling of IncRNAs in C3H10T1/2 mesenchymal stem cells undergoing early osteoblast differentiation
}

\author{
CHANGQING ZUO ${ }^{1}$, ZONGGUI WANG ${ }^{2,3,4}$, HANYUN LU $^{1}$, ZHONG DAI $^{1}$, XINGUANG LIU $^{2,3,4}$ and LIAO CUI ${ }^{1}$ \\ Departments of ${ }^{1}$ Pharmacology, ${ }^{2}$ Biochemistry and Molecular Biology, ${ }^{3}$ Institute of Aging Research, \\ Guangdong Medical College; ${ }^{4}$ Guangdong Provincial Key Laboratory of Medical Molecular Diagnostics, \\ Dongguan, Guangdong 523808, P.R. China
}

Received January 24, 2013; Accepted June 4, 2013

DOI: $10.3892 / \mathrm{mmr} .2013 .1540$

\begin{abstract}
Protein-coding genes and small non-coding microRNAs involved in the guidance of differentiation in mesenchymal stem cells (MSCs) into osteoblasts have been extensively investigated in previous studies. However, long non-coding RNAs (lncRNAs), which account for a large proportion of the genomic sequences in numerous species, have not yet been reported. In the present study, the lncRNA expression profile was analyzed using the Arraystar lncRNA array in C3H10T1/2 MSCs undergoing early osteoblast differentiation and 116 differentially expressed lncRNAs were identified between BMP-2 treated and untreated groups. Among these IncRNAs, 59 were upregulated and 57 were downregulated in BMP-2 treated groups. In addition, 24 cooperatively differentially expressed lncRNAs and nearby mRNA pairs were found. For example, mouselincRNA0231 and its nearby gene, EGFR, were downregulated, while lncRNA NR_027652 and its nearby gene, DLK1, were upregulated. These observations may be part of the regulatory mechanisms of IncRNAs in the control of osteoblast differentiaton. In conclusion, results of the present study indicate that lncRNA expression profiles are significantly altered in $\mathrm{C} 3 \mathrm{H} 10 \mathrm{~T} 1 / 2$ undergoing early osteoblast differentiation and these results may provide insight into the mechanisms responsible for osteoblast differentiation.
\end{abstract}

\section{Introduction}

Osteoblast differentiation from mesenchymal stem cells (MSC) is a highly regulated process guided by complex signaling cascades. BMP-2 functions as an effective osteo-

Correspondence to: Dr Changqing Zuo, Department of Pharmacology, Guangdong Medical College, 1 Xincheng Avenue, Songshan Lake Industrial and Technology Park, Dongguan, Guangdong 523808, P.R. China

E-mail:wsgwz77@gmail.com

Key words: long non-coding RNA, osteoblast differentiation, mesenchymal stem cells, bioinformatics blast-inducing signal and has been investigated extensively (1). In the past two decades, a number of transcription factors and small non-coding microRNAs involved in BMP-2-induced osteoblast differentiation have been identified (2-5). However, the precise molecular mechanisms of osteoblast differentiation remain largely unknown.

Long non-coding RNAs (long ncRNAs or lncRNAs) are generally considered to represent non-protein coding transcripts of $>200$ nucleotides (6). An increasing number of studies have reported that lncRNAs participate in diverse biological processes through distinct mechanisms in mammalian biology $(7,8)$. Aberrant lncRNA expression and mutations have been linked to a diverse number of human diseases, including cancer, cardiovascular dieases and Alzheimer's disease (9-11). Specifically, findings of previous studies demonstrated that IncRNAs are extremely important for the control of cell or tissue differentiation (12-15). Although an increasing number of functional lncRNAs have been characterized thus far, the functions of the majority of lncRNAs remain unknown (16).

It is not known whether MSC commitment and differentiation into osteoblasts relies on the modulation of lncRNA expression. To address this question, in the present study, lncRNA expression profiling was performed in MSCs undergoing differentiation into osteoblasts at day 1 and 4 . Differentially expressed lncRNAs were then selected for bioinformatic analyses. Results of this study are likely to provide an important foundation for future studies on the lncRNA modulation of osteoblastic differentiation.

\section{Materials and methods}

Cell culture and osteoblast differentiation. C3H10T1/2 cells were obtained from the Chinese Academy of Science Cell Bank (Shanghai, China) and were cultured in Dulbecco's modified Eagle's medium (DMEM) containing 10\% fetal bovine serum (FBS; both Hyclone Laboratories, Inc., Logan, UT, USA) at $37^{\circ} \mathrm{C}$ in a humidified atmosphere of $5 \% \mathrm{CO}_{2}$ in air. To induce osteoblast differentiation, the medium was replaced with low-serum medium, consisting of DMEM supplemented with 5\% FBS and $200 \mathrm{ng} / \mathrm{ml} \mathrm{rhBMP}-2$ (R\&D Systems, Minneapolis, MN, USA) and the medium was changed every 2-3 days. 
A

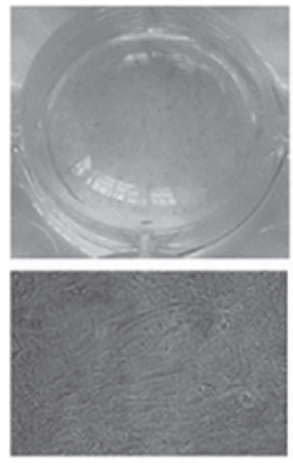

control

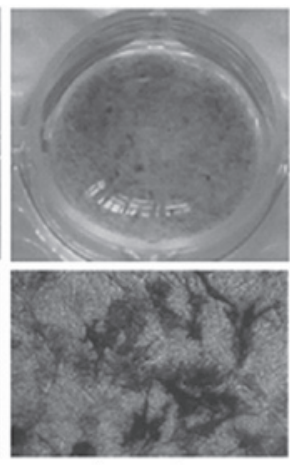

bmp-2
B

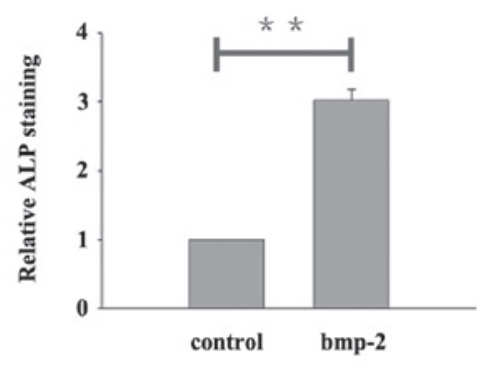

Figure 1. ALP staining for C3H10T1/2 mesenchymal stem cell osteoblast differentiation after 7 days of treatment with and without BMP-2. ${ }^{* *}$ P $<0.05$ compared to the control. (A and B) A significant increase in ALP acitivity in BMP-2-induced C3H10T1/2 cells was observed, indicating that BMP-2 stimulates C3H10T1/2 cells into early osteoblast differentiation.

Alkaline phosphatase (ALP) staining. Levels of osteoblast differentiation in $\mathrm{C} 3 \mathrm{H} 10 \mathrm{~T} 1 / 2$ cells were determined using ALP staining. For ALP staining, cells were washed with PBS twice, fixed with $70 \%$ ethanol for $20 \mathrm{~min}$, rinsed three times with deionized water and then incubated with the BCIP/NBT liquid substrate system (Sigma-Aldrich, St. Louis, MO, USA), an ALP substrate solution, for $30 \mathrm{~min}$. Images of the stained cells were then captured. For quantitative analysis, the ALP stain was extracted with $10 \%$ cetylpyridinium chloride for $15 \mathrm{~min}$ and quantified by measuring its absorbance at $540 \mathrm{~nm}$. Relative ALP staining was then calculated as a fold change of the control.

RNA isolation. Following incubation with $200 \mathrm{ng} / \mathrm{ml}$ BMP-2 for 1 or 4 days, total RNA was extracted using TRIzol (Invitrogen Life Technologies, Carlsbad, CA, USA). RNA was also extracted from BMP-2 untreated cells. Total RNA from each sample was quantified using the NanoDrop 1000 spectrophotometer (Thermo Fisher Scientific, Waltham, MA, USA) and RNA integrity was assessed using standard denaturing agarose gel electrophoresis.

Microarray detection and analysis. For microarray analysis, the Agilent Array platform (Agilent Technologies, Santa Clara, CA, USA) was employed. Each sample was amplified and transcribed into fluorescent cRNA along the entire length of the transcripts without 3' bias utilizing a random priming method (Quick Amp Labeling kit, One-Color, Agilent Technologies, p/n 5190-0442). The labeled cRNAs were hybridized onto the Mouse lncRNA Array v2.0 (8x60K; Arraystar Inc., Rockville, MD, USA) which was designed for the global profiling of 31,423 mouse IncRNAs and 25,376 protein-coding transcripts. The slides were washed and the arrays were scanned using the Agilent G2505C microarray scanner. Agilent Feature Extraction software (version 11.0.1.1) was used to analyze the acquired array images. Quantile normalization and subsequent data processing were performed using the GeneSpring GX version 11.5.1 software package (Agilent Technologies). Differentially expressed IncRNAs and mRNAs were identified through fold change filtering.

Category analysis of differentially expressed lncRNAs. According to lncRNA genomic locations relative to protein coding genes, lncRNAs are categorized as: i) sense, ii) antisense, iii) bidirectional, iv) intronic and v) intergenic (17). The differentially expressed lncRNAs identified in this study were categorized based on these groupings.

Bioinformatic analysis of $\operatorname{lnc} R N A$ relative to nearby protein-coding genes. One important function of lncRNAs is to regulate the expression of nearby protein-coding genes. Therefore, protein-coding genes were searched for differentially expressed lncRNAs using the UCSC Genome Browser (http://genome.ucsc.edu/cgi-bin/hgGateway). Genes transcribed within $300 \mathrm{~kb}$ were considered to represent nearby coding genes and predicted lncRNAs nearby these coding genes were integrated with differentially expressed mRNA in the microarray (fold change $\geq 2.0$ at day 4) (18). The regulatory network between IncRNAs and nearby coding genes was visually presented using the Cytoscape program (http://www. cytoscape.org/).

Statistical analysis. Experiments were repeated three times with the exception of microarray experiments. Two group comparisons were performed using the Student's t test. $\mathrm{P}<0.05$ was considered to indicate a statistically significant difference.

\section{Results}

BMP-2 increased osteoblast-specific marker ALP activity. Osteoblast differentiation from MSCs is regulated by various cytokines and growth factors. BMP-2, a transforming growth factor $\beta$ superfamily member, is well known as one of the most powerful osteoblast promoting factors (19). ALP activity is an early osteoblast differentiation marker. In the present study, ALP activity in C3H10T1/2 cells with and without BMP-2 treatment was analyzed for 7 days to investigate BMP-2-induced C3H10T1/2 cell osteoblast differentiation. A significant increase in ALP acitivity in BMP-2-induced C3H10T1/2 cells was observed (Fig. 1), indicating that BMP-2 stimulates C3H10T1/2 cells into early osteoblast differentiation.

Differentially expressed profiles of the lncRNAs in BMP-2induced C3H1OTI/2 cell osteoblast differentiation. To investigate the expression profiles of lncRNAs during MSC osteoblast differentiation, total RNA was extracted from 
Table I. Upregulated expression of 59 lncRNAs in BMP-2 treated C3H10T1/2 cells for 1 and 4 days.

\begin{tabular}{|c|c|c|}
\hline lncRNA symbol/name & lncRNA symbol/name & lncRNA symbol/name \\
\hline uc009odu.1 & uc009gzn.1 & uc009bxq.1 \\
\hline uc008drt.1 & AK144695 & ВC023483 \\
\hline uc007djg.1 & uc007cyp.1 & NR_027652 \\
\hline CB272499 & AV570737 & AK201409 \\
\hline AK043290 & AK032849 & AK032137 \\
\hline AK030568 & mKIAA0031 & NR_033578 \\
\hline AK148154 & uc.77 & AK148935 \\
\hline AA656495 & uc007rco.1 & ENSMUST00000122262 \\
\hline ENSMUST00000122215 & ENSMUST00000121177 & ENSMUST00000170943 \\
\hline ENSMUST00000120905 & ENSMUST00000120431 & ENSMUST00000120163 \\
\hline ENSMUST00000120127 & ENSMUST00000120017 & ENSMUST00000118988 \\
\hline ENSMUST00000118701 & ENSMUST00000118061 & ENSMUST00000117850 \\
\hline ENSMUST00000074110 & ENSMUST00000053965 & ENSMUST00000172364 \\
\hline ENSMUST00000171670 & ENSMUST00000119457 & ENSMUST00000080271 \\
\hline ENSMUST00000165702 & ENSMUST00000135189 & ENSMUST00000163579 \\
\hline ENSMUST00000160955 & ENSMUST00000153890 & ENSMUST00000151866 \\
\hline ENSMUST00000170806 & ENSMUST00000170714 & ENSMUST00000143202 \\
\hline ENSMUST00000121036 & ENSMUST00000119775 & ENSMUST00000081067 \\
\hline ENSMUST00000126138 & MM9LINCRNAEXON11209- & MM9LINCNAEXON10436+ \\
\hline MM9LINCRNAEXON11971- & MM9LINCRNAEXON11966- & \\
\hline
\end{tabular}

lncRNAs, long non-coding RNAs.

A

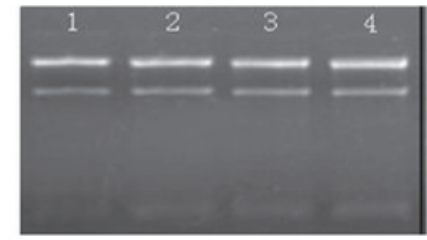

Lane 1 (DZ1): BMP-2 untreated 1 day Lane 2 (SY1): BMP-2 treated 1 day Lane 3 (DZ4): BMP-2 untreated 4 day Lane 4 (SY4): BMP-2 treated 4 day
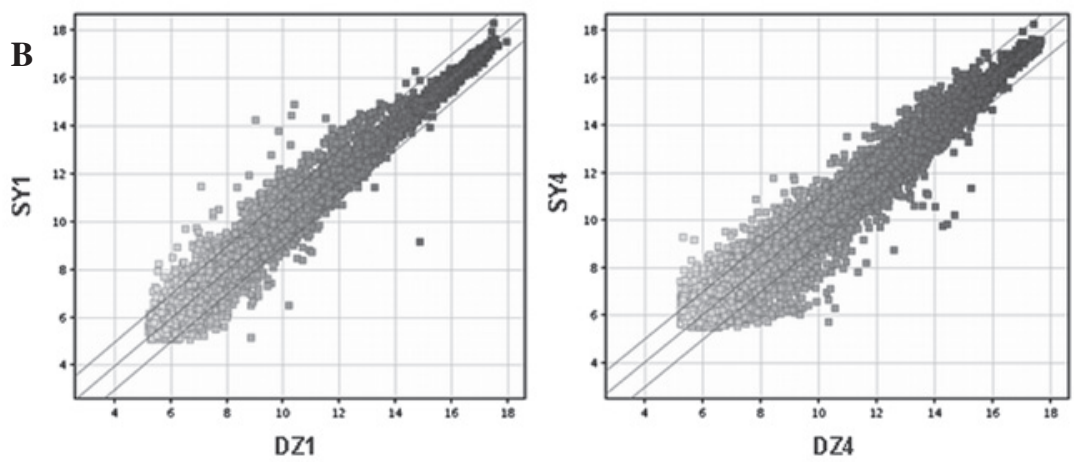

Figure 2. (A) Gel electrophoresis was used to determine the quality of total RNA. (B) Scatter plot analysis of the microarray data between BMP-2-treated and untreated cells.

BMP-2 treated and untreated cells at day 1 and $4 . \mathrm{OD}_{260 / 280}$ ratios were close to 2.0 and $\mathrm{OD}_{260 / 230}$ ratios were $>2.0$ for all the samples. The quality of total RNA was checked by gel electrophoresis (Fig. 2A), confirming that the RNA was of good quality. Scatter plot analysis of the microarray data is shown in Fig. 2B. Microarray expression analysis of lncRNAs was then performed.Firstly, IncRNAs upregulated or downregulated by $>1.5$-fold in BMP-2 treated or untreated C3H10T1/2 cells for 1 day were screened, revealing 886 upregulated and 825 downregulated lncRNAs in the BMP-2 treated group. Secondly, 595 upregulated and 548 downregulated lncRNAs by $>2$-fold were identified at day 4. Continuously upregulated or downregulated lncRNAs following prolonged BMP-2 treatment may be more important for the control of osteoblast differentiation. We also identified 59 upregulated (Table I) and 57 downregulated lncRNAs (Table II) following 1 and 4 days of BMP-2 treatment in accordance with the described thresholds.

Category analysis of differentially expressed lncRNAs. Based on a previous study by Ponting et al (17), of the 116 differentially expressed lncRNAs identified in this study, $60.3 \%$ were categorized as intergenic. In addition, $20.7 \%$ were catagorized as sense. Details of the categories are presented in Table III.

Bioinformatic analysis of IncRNAs relative to osteoblast differentiation. Unlike proteins or microRNAs, IncRNA function cannot be inferred from sequence or structure. Studies have hypothesized a number of regulatory paradigms to explain 
Table II. Downregulated expression of 57 lncRNAs in BMP-2 treated C3H10T1/2 cells for 1 and 4 days.

\begin{tabular}{lll}
\hline lncRNA symbol/name & lncRNA symbol/name & lncRNA symbol/name \\
\hline uc009rnr.1 & uc009qfj.1 & BC099572 \\
AK044623 & mD53 & uc007cpz.1 \\
BC038927 & AK142678 130026 I21Rik \\
BC038927 & AK137923 & mouselincRNA0231 \\
mouselincRNA0106 & mouselincRNA0243 & mouselincRNA1280 \\
AK043049 & BM942986 & AK156713 \\
AK143058 & AK137040 & AK136357 \\
AK134233 & AK089560 & AK054249 \\
AK046177 & AK044187 & AK043294 \\
AK039250 & AK032775 & AK031656 \\
AK031010 & AK030271 & AK029300 \\
AK020801 & AK014853 & AK013539 \\
AI551087 & ENSMUST00000125230 & ENSMUST00000122459 \\
ENSMUST00000116393 & ENSMUST00000170072 & ENSMUST00000165050 \\
ENSMUST00000162276 & ENSMUST00000160831 & ENSMUST00000160674 \\
ENSMUST00000156260 & ENSMUST00000154798 & ENSMUST00000148528 \\
ENSMUST00000147939 & ENSMUST00000137032 & ENSMUST00000133270 \\
ENSMUST00000129933 & MM9LINCRNAEXON10823+ & MM9LINCRNAEXON11750+ \\
MM9LINCRNAEXON11996+ & MM9LINCRNAEXON11765+ & MM9LINCRNAEXON11642-
\end{tabular}

lncRNAs, long non-coding RNAs.

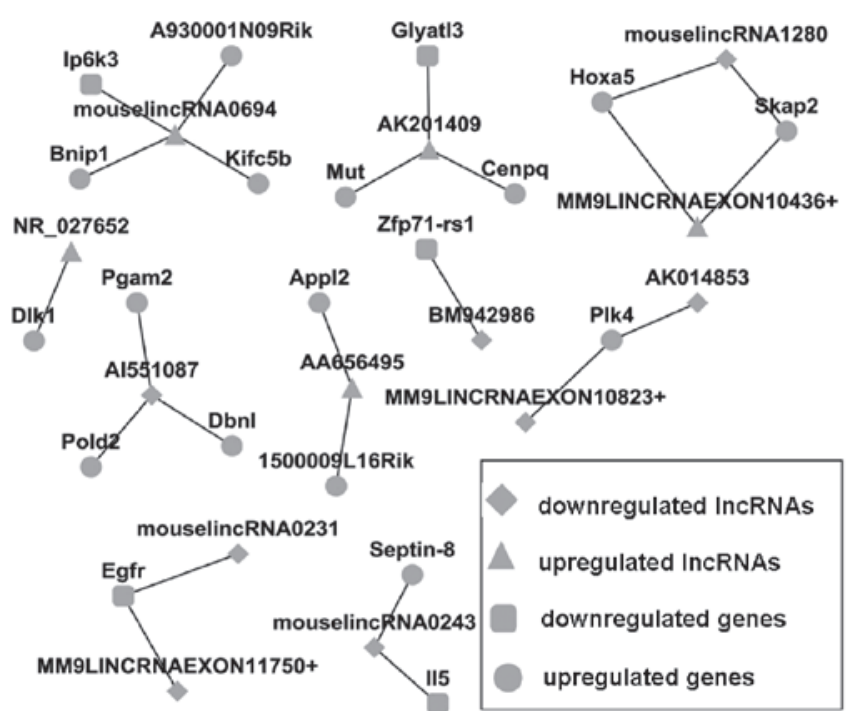

Figure 3. Regulatory network between differentially expressed lncRNAs and nearby protein-coding genes pairs. lncRNAs, long non-coding RNAs.

the mechanism by which lncRNAs function. In the present study, the genomic context of lncRNAs was highlighted. Differentially expressed nearby mRNAs were combined and 24 differentially expressed lncRNAs and nearby coding genes pairs were identified for 13 differentially expressed lncRNAs and 20 differentially expressed mRNAs. Using the Cytoscape program, a regulatory network was constructed between differentially expressed lncRNA and nearby coding genes (Fig. 3).
Table III. Category analysis of differentially expressed lncRNAs.

\begin{tabular}{lccr}
\hline & \multicolumn{2}{c}{$\operatorname{lncRNAs}$} \\
\cline { 2 - 3 } Category & Upregulated & Downregulated & $\%$ \\
\hline Sense & 14 & 10 & 20.7 \\
Antisense & 7 & 12 & 16.4 \\
Bidirectional & 2 & 1 & 2.6 \\
Intronic & 0 & 0 & 0.0 \\
Intergenic & 36 & 24 & 60.3 \\
\hline
\end{tabular}

lncRNAs, long non-coding RNAs.

\section{Discussion}

Specific lncRNAs, including linc-MD1, TINCR and ANCR, have been previously reported to be involved in the control of cell or tissue differentiation (12-15). In the present study, the Arraystar microarray analysis was used to identify differentially expressed lncRNAs associated with BMP-2 stimulated osteoblast differentiation. To the best of our knowledge, this is the first study to demonstrate genome-wide differentially expressed lncRNA profiling in MSCs undergoing early osteoblast differentiation.

Although lncRNAs may have an important impact on a diverse range of human diseases, the current understanding of the molecular mechanisms by which lncRNAs function remain largely unknown. Previous studies have demonstrated 
that lncRNAs may function by controlling the transcriptional regulation of neighboring coding genes $(7,17,20)$. For example, the ncRNA, Evf2, forms a complex with the transcription factor, Dlx2, to induce the expression of adjacent proteincoding genes (21). Identifying differentially expressed nearby coding mRNA may enhance understanding of the function and potential regulatory mechanisms for lncRNAs. In the current study, 24 differentially expressed lncRNAs and nearby coding genes pairs were identified. Among the regulatory network between differentially expressed lncRNAs and nearby protein-coding genes pairs, specifc nodes have been previously reported to be involved in osteoblast differentiation or bone metabolism. For example, the protein-coding gene, EGFR, a nearby coding gene for differentially expressed lncRNA, mouselincRNA0231, was downregulated by 2.2and 2.8-fold following BMP-2 treatment for 1 and 4 days, respectively. A previous study reported that EGFR signaling suppresses osteoblast differentiation by inhibiting the expression of master osteoblastic transcription factors, Runx 2 and Osterix (22). We hypothesized that mouselincRNA0231 may negatively regulate osteoblast differentiation by affecting EGFR signaling. DLK1 is a novel regulator of bone mass and inhibits bone formation and stimulates bone resorption $(23,24)$ and was upregulated in the BMP-2 treatment group. This upregulation may have reduced the action of BMP-2. DLK1 is a nearby coding gene for differentially expressed lncRNA NR_027652. Therefore, a synergistic effect on osteoblast differentiation may exist between DLK1 and NR_027652. IL-5, is a nearby coding gene for mouselincRNA0243 and is a $\mathrm{T}$ cell-derived factor. Macias et al previously reported that overexpression of IL-5 in a transgenic mouse line mediated bone formation through the mobilization of marrow-derived osteogenic progenitors (25). These pairings between lncRNA and nearby coding protein may represent one of the regulatory mechanisms by which lncRNAs control osteoblast differentiaton through the regulation of neighboring osteoblast-related gene expression. However, further studies must be performed to prove this hypothesis.

In conclusion, 116 continuously differentially expressed lncRNAs were identified in this study during BMP-2-stimulated osteoblast differentiation for 1 and 4 days in C3H10T1/2 mensenchymal stem cells. In addition, potential regulatory mechanisms by which IncRNAs control osteoblast differentiation were identified by bioinformatic analysis. Although more studies are required to demonstrate the precise role and mechanisms of lncRNAs in osteoblast differentiation, lncRNAs appear to be potent candidates for osteoblast differentiation or therapeutic agents for osteogenic disorders in the future.

\section{Acknowledgements}

This study was supported by grants from the National Natural Science Foundation of China (nos. 81101357 and 81170327), the Science and Technological Program for Dongguan's Higher Education, Science and Research and Health Care Institutions (no. 2011108102029) and the Science and Technology Innovation Fund of Guangdong Medical College (no. STIF201104). Microarray experiments were performed by KangChen Bio-tech (Shanghai, China).

\section{References}

1. Wozney JM, Rosen V, Celeste AJ, et al: Novel regulators of bone formation: molecular clones and activities. Science 242. 1528-1534, 1988 .

2. Lin GL and Hankenson KD: Integration of BMP, Wnt and notch signaling pathways in osteoblast differentiation. J Cell Biochem 112: 3491-3501, 2011.

3. Huang J, Zhao L, Xing L and Chen D: MicroRNA-204 regulates Runx 2 protein expression and mesenchymal progenitor cell differentiation. Stem Cells 28: 357-364, 2010.

4. Rosen V: BMP2 signaling in bone development and repair Cytokine Growth Factor Rev 20: 475-480, 2009.

5. Dong S, Yang B, Guo H and Kang F: MicroRNAs regulate osteogenesis and chondrogenesis. Biochem Biophys Res Commun 418: 587-591, 2012.

6. ENCODE ProjectConsortium; BirneyE,Stamatoyannopoulos JA, Dutta A, et al: Identification and analysis of functional elements in $1 \%$ of the human genome by the ENCODE pilot project. Nature 447: 799-816, 2007.

7. Hung T and Chang HY: Long noncoding RNA in genome regulation: prospects and mechanisms. RNA Biol 7: 582-585, 2010.

8. Wilusz JE, Sunwoo H and Spector DL: Long noncoding RNAs: functional surprises from the RNA world. Genes Dev 23: 1494-1504, 2009.

9. Huarte M, Guttman M, Feldser D, et al: A large intergenic noncoding RNA induced by p53 mediates global gene repression in the p53 response. Cell 142: 409-419, 2010.

10. Esteller M: Non-coding RNAs in human disease. Nat Rev Genet 12: 861-874, 2011.

11. Sui W, Li H, Ou M, Tang D and Dai Y: Altered long non-coding RNA expression profile in patients with IgA-negative mesangial proliferative glomerulonephritis. Int J Mol Med 30: 173-178, 2012.

12. Cesana M, Cacchiarelli D, Legnini I, et al: A long noncoding RNA controls muscle differentiation by functioning as a competing endogenous RNA. Cell 147: 358-369, 2011.

13. Kretz M, Siprashvili Z, Chu C, et al: Control of somatic tissue differentiation by the long non-coding RNA TINCR. Nature 493: 231-235, 2013.

14. Kretz M, Webster DE, Flockhart RJ, et al: Suppression of progenitor differentiation requires the long noncoding RNA ANCR. Genes Dev 26: 338-343, 2012.

15. Ng SY, Johnson R and Stanton LW: Human long non-coding RNAs promote pluripotency and neuronal differentiation by association with chromatin modifiers and transcription factors. EMBO J 31: 522-533, 2011.

16. Wang KC and Chang HY: Molecular mechanisms of long noncoding RNAs. Mol Cell 43: 904-914, 2011.

17. Ponting CP, Oliver PL and Reik W: Evolution and functions of long noncoding RNAs. Cell 136: 629-641, 2009.

18. Han L, Zhang K, Shi Z, et al: LncRNA profile of glioblastoma reveals the potential role of IncRNAs in contributing to glioblastoma pathogenesis. Int J Oncol 40: 2004-2012, 2012.

19. Chen D, Zhao M and Mundy GR: Bone morphogenetic proteins. Growth Factors 22: 233-241, 2004

20. Mercer TR, Dinger ME and Mattick JS: Long non-coding RNAs: insights into functions. Nat Rev Genet 10: 155-159, 2009.

21. Feng J, Bi C, Clark BS, Mady R, Shah P and Kohtz JD: The Evf-2 noncoding RNA is transcribed from the Dlx-5/6 ultraconserved region and functions as a Dlx-2 transcriptional coactivator. Genes Dev 20: 1470-1484, 2006.

22. Zhu J, Shimizu E, Zhang X, Partridge NC and Qin L: EGFR signaling suppresses osteoblast differentiation and inhibits expression of master osteoblastic transcription factors Runx2 and Osterix. J Cell Biochem 112: 1749-1760, 2011.

23. Abdallah BM, Ding M, Jensen $\mathrm{CH}$, et al: Dlk1/FA1 is a novel endocrine regulator of bone and fat mass and its serum level is modulated by growth hormone. Endocrinology 148: 3111-3121, 2007.

24. Abdallah BM, Ditzel N, Mahmood A, et al: DLK1 is a novel regulator of bone mass that mediates estrogen deficiency-induced bone loss in mice. J Bone Miner Res 26: 1457-1471, 2011.

25. Macias MP, Fitzpatrick LA, Brenneise I, McGarry MP, Lee JJ and Lee NA: Expression of IL-5 alters bone metabolism and induces ossification of the spleen in transgenic mice. J Clin Invest 107: 949-959, 2001. 